\title{
Making Mistakes Saves the Single Observer's World of the Extended Wigner's Friend Experiment
}

\author{
Szymon tukaszyk \\ Łukaszyk Patent Attorneys, Katowice, Poland \\ Email: szymon@patent.pl
}

How to cite this paper: Łukaszyk, S. (2022) Making Mistakes Saves the Single Observer's World of the Extended Wigner's Friend Experiment. Journal of Quantum Information Science, 12, 1-12. https://doi.org/10.4236/jqis.2022.121001

Received: January 16, 2022

Accepted: February 20, 2022

Published: February 23, 2022

Copyright ( 2022 by author(s) and Scientific Research Publishing Inc. This work is licensed under the Creative Commons Attribution International License (CC BY 4.0).

http://creativecommons.org/licenses/by/4.0/ (c) (i) Open Access

\begin{abstract}
The Extended Wigner's Friend thought experiment, comprising a quantum system containing an agent who draws conclusions upon observing the outcome of a measurement of a quantum state prepared in two nonorthogonal versions by another agent, led its authors to conclude that quantum theory cannot consistently describe the use of itself. It has also been proposed that this thought experiment is equivalent to entangled state (Bell-type) experiments. It is argued in this paper that the assumption of the freedom of choice of the first Wigner's friend regarding how to prepare a quantum state in one of the two available nonorthogonal versions invalidates such equivalence.
\end{abstract}

\section{Keywords}

Measurement Problem, Wigner's Friend, Bell's Theorem, Observer-Independent Facts, Quantum Contextuality, Freedom of Choice

\section{Introduction}

The Extended Wigner's Friend (EWF) thought experiment contains a contradiction, which initially led its authors to give up the view that there is one single world [1], and later to conclude that Quantum Theory (QT) cannot consistently describe the use of itself [2], as the contradiction appeared within a single world. Indeed, many-worlds interpretation of QT has paradoxical features of its own [3] and is not only counterfactually indefinite but also factually indefinite [4]. On the other hand, if QT cannot consistently describe the use of itself, it would be an ultimate theory for the perceived nature, since no consistent system of axioms can demonstrate its own consistency, which is the conclusion of the $2^{\text {nd }}$ Gödel's incompleteness theorem, if only the Peano axioms of arithmetic are universally valid 
(i.e., as long as the perceived nature is subject to these axioms).

It is, however, questionable how QT is used in the EWF to describe the use of itself. The EWF is allegedly not just a thought experiment. Its authors claim, for example, that if it were implemented as a game between a gambler and a casino, both parties would likely have entered into a dispute, putting forward contradicting assertions based on quantum-mechanical reasoning. These assertions would have to be accepted as two alternative (observer-dependent) facts about what was the result of the first measurement in this thought experiment [2].

The paper aims to evaluate the prospects of the EWF implementation as a casino game. The feasibility of implementing Bell-Wigner type experiments (e.g., [5] [6] [7] [8]) involving entangled states, as casino games is also discussed.

\section{The EWF with a Super-Observer}

In each round of the EWF, in her sealed lab Alice prepares a first state (a qubit):

$$
|\alpha\rangle=\sqrt{\frac{1}{3}}|h\rangle+\sqrt{\frac{2}{3}}|t\rangle,
$$

measures it at a certain time $t_{0}$ in a basis $\{|h\rangle,|t\rangle\}$, records the measurement, and prepares a second state:

$$
|\beta\rangle=\left\{\begin{array}{ll}
|0\rangle & \text { iff }|\alpha\rangle=|h\rangle \\
\sqrt{\frac{1}{2}}(|0\rangle+|1\rangle)=|+\rangle & \text { iff }|\alpha\rangle=|t\rangle
\end{array} .\right.
$$

Alice hands then the second state ${ }^{1}$ to Bob (2) residing in another sealed lab. At a later time $t_{1}>t_{0}$, Bob measures the received second state in a basis $\{|0\rangle,|1\rangle\}$. At even later time $t_{2}>t_{1}$ Charlie measures the first state (1) emitted from Alice's lab in a first Hadamard basis defined as:

$$
|\square\rangle \doteq \sqrt{\frac{1}{2}}(|h\rangle+|t\rangle), \quad|O\rangle \doteq \sqrt{\frac{1}{2}}(|h\rangle-|t\rangle),
$$

and the second state (2) emitted from Bob's lab in a second Hadamard defined as:

$$
|+\rangle \doteq \sqrt{\frac{1}{2}}(|0\rangle+|1\rangle), \quad|-\rangle \doteq \sqrt{\frac{1}{2}}(|0\rangle-|1\rangle),
$$

and the round is completed. Since Charlie does not know the measurement results of the first (1) and the second (2) states emitted from Alice's and Bob's labs, statistics of Charlie's measurements of the first state (1) in a large number of rounds of this experiment can be described by the mixed state density matrix:

$$
\rho_{\alpha}=\frac{1}{3}|h\rangle\left\langle h\left|+\frac{2}{3}\right| t\right\rangle\langle t|,
$$

while statistics of Charlie's measurements of the second state (2), by the mixed state density matrix:

${ }^{1}$ Describing (gruesomely) this preparation process: Alice puts a cat into a Schrödinger's box provided with all the necessary equipment (a Geiger counter, a flask of poison, etc.), if she measures $|t\rangle$, but she poisons the cat before putting its corpse into the box, if she measures $|h\rangle$. She delivers the box to Bob, who will open it to find out whether the cat is alive $(|1\rangle)$ or poisoned $(|0\rangle)$. 


$$
\rho_{\beta}=\frac{1}{3}|0\rangle\left\langle 0\left|+\frac{2}{3}\right|+\right\rangle\langle+|,
$$

which has purity $\operatorname{Tr}\left(\rho_{\beta}^{2}\right)=7 / 9$ larger than purity $\operatorname{Tr}\left(\rho_{\beta^{\prime}}^{2}\right)=5 / 9$ of the density matrix:

$$
\rho_{\beta^{\prime}}=\frac{2}{3}|0\rangle\left\langle 0\left|+\frac{1}{3}\right| 1\right\rangle\langle 1|,
$$

that would yield the same measurement probabilities.

There is nothing contradicting (as nothing is entangled) in this setup. Measurement probabilities for a large number of rounds are nonzero

$$
\begin{aligned}
& p(|h\rangle)=p(|1\rangle)=1 / 3, \quad p(|t\rangle)=p(|0\rangle)=2 / 3 ; \quad p(|\square\rangle)=p(|\bigcirc\rangle)=1 / 2 ; \\
& p(|+\rangle)=5 / 6, \quad p(|-\rangle)=1 / 6 .
\end{aligned}
$$

Due to the principle of locality, however, this kind of thought experiments containing a qubit and someone who measures this qubit contained in a box or a lab isolated from the environment (Schrödinger's cat, Wigner's friend [9], Deutsch's variant [10], and the EWF) are described as coherent big quantum states of those boxes or labs. In the case of Alice's lab, for example, this big quantum state is described as a tensor product of the basic quantum state (1), of some device that enables for a measurement of this basic state and finally of Alice herself in her lab including her sense organs, her brain, etc. This indeed seems unrealistic, even if not explicitly precluded by the laws of QT as such. Schrödinger's cat has nothing to do with quantum information science, even if the latter can be harnessed to kill the cat. Deutsch's variant [10], in which a friend informs Wigner that she has a definite measurement result, but does not reveal this result, so as not to accidentally destroy the superposition of the big quantum state from inside of the lab, is particularly instructive.

Even if the assumptions (Q, C, S) of the EWF do not explicitly include locality [7], locality is used in the EWF [11] to model the enclosed immediate surroundings of the Alice's and Bob's labs including Alice and Bob themselves and their actions in space and time, as a coherent, entangled big quantum state that can be defined [12]; by Charlie as:

$$
|\psi\rangle_{A B}=\sqrt{\frac{1}{3}}|h\rangle|0\rangle+\sqrt{\frac{1}{3}}|t\rangle|0\rangle+\sqrt{\frac{1}{3}}|t\rangle|1\rangle,
$$

after excluding Alice, Bob and their devices subsystems states whose exact properties do not need to be specified ([5], p. 16). They correspond to the definitemeasurement-result information in Deutsch's variant [10] of the Wigner's friend [9] thought experiment, and are the same, regardless of the measurement result. Nonmaximally entangled state (8) shows that unlike Schrödinger's cat, Wigner's friend, or its Deutsch's variant, only the EWF pretends to be a Bell type experiment.

In Charlie's bases $\{|\square\rangle,|O\rangle\}$ and $\{|+\rangle,|-\rangle\}$ the state (8) is:

$$
\begin{aligned}
|\psi\rangle_{C} & =\sqrt{\frac{9}{12}}|\square\rangle|+\rangle+\sqrt{\frac{1}{12}}|\square\rangle|-\rangle-\sqrt{\frac{1}{12}}|O\rangle|+\rangle+\sqrt{\frac{1}{12}}|O\rangle|-\rangle \\
& =\sqrt{\frac{9}{12}}|\square\rangle|+\rangle+\sqrt{\frac{1}{12}}|\square\rangle|-\rangle-\sqrt{\frac{2}{12}}|O\rangle|1\rangle
\end{aligned}
$$


and in mixed bases $\{|h\rangle,|t\rangle\},\{|+\rangle,|-\rangle\} ;\{|\square\rangle,|O\rangle\},\{|0\rangle,|1\rangle\}$ it is:

$$
\begin{gathered}
|\psi\rangle_{A C}=\sqrt{\frac{1}{6}}|h\rangle|+\rangle+\sqrt{\frac{1}{6}}|h\rangle|-\rangle+\sqrt{\frac{4}{6}}|t\rangle|+\rangle=\sqrt{\frac{1}{3}}|h\rangle|0\rangle+\sqrt{\frac{2}{3}}|t\rangle|+\rangle, \\
|\psi\rangle_{C B}=\sqrt{\frac{4}{6}}|\square\rangle|0\rangle+\sqrt{\frac{1}{6}}|\square\rangle|1\rangle-\sqrt{\frac{1}{6}}|O\rangle|1\rangle=\sqrt{\frac{2}{3}}|\square\rangle|0\rangle+\sqrt{\frac{1}{3}}|t\rangle|1\rangle .
\end{gathered}
$$

The following simple argumentation used to expose the contradiction of the EWF [12] is similar to the one used in Ref. [13] to illustrate the mystery of the quantum cakes ("a simple 'real-world' explanation of the proof of quantum mechanical nonlocality without the use of inequalities"; I will further call it "quantum-cakes explanation"):

(\#1) We know from $|\psi\rangle_{C}(9)$ that measurements of $|O\rangle$ and $|-\rangle$ are possible with probability $p=1 / 12$;

(\#2) We know from $|\psi\rangle_{A C}(10)$ that $|-\rangle \Rightarrow|h\rangle^{2}$;

(\#2') We know from $|\psi\rangle_{C B}(11)$ that $|O\rangle \Rightarrow|1\rangle$;

(\#3) We know from $|\psi\rangle_{A B}(8)$ that $|h\rangle \Rightarrow|0\rangle$.

(In other words $\left|O_{-}\right\rangle \Rightarrow|h 1\rangle \cap|h\rangle \Rightarrow|0\rangle$, which is contradicting).

We note in passing that the state (8) in bases (3), (4) is more symmetric than the oven state and bases used in Ref. [13], and that the concept of time is irrelevant in the quantum-cakes explanation.

The EWF contradiction is, however, derived in Ref. [2] on the grounds of the following predictions made by Alice, Bob, and Charlie in different times $t_{0}, t_{1}$, and $t_{2}$ during a happy round of this experiment. Each round goes like this:

(0) Alice and Bob prepare and/or measure their states (1) and (2) according to the procedure described in the outset, and Charlie measures $|\psi\rangle_{C}$, that describes the big quantum state of both Alice's and Bob's labs (at time $t_{2}$ );

: $:$ If Charlie's measurement result is $|\square+\rangle,|\square-\rangle$, or $|\bigcirc+\rangle$, the round is completed with no contradiction, and a new round begins;

() If Charlie's measurement result is $\left|O^{-}\right\rangle$, then they have a happy round and

(A) Alice knows from (2) that $|t\rangle$ (at time $\left.t_{0}\right) \Rightarrow|+\rangle$ (at time $t_{2}$ );

(B) Bob knows from (2) that $|1\rangle$ (at time $\left.t_{1}\right) \Rightarrow|t\rangle$ (at time $t_{0}$ );

(C) Charlie knows from $|\psi\rangle_{C}(9)$ that $|O\rangle$ (at time $\left.t_{2}\right) \Rightarrow|1\rangle$ (at time $t_{1}$ ).

All these four conditions, in a happy round of the EWF, also contradict each other: if $\odot$ then $|O\rangle \Rightarrow|1\rangle(\mathrm{C}),|1\rangle \Rightarrow|t\rangle(\mathrm{B})$, and $|t\rangle \Rightarrow|+\rangle$ (A) (In other words if () then $\mathrm{C} \Rightarrow \mathrm{B} \cap \mathrm{B} \Rightarrow \mathrm{A} \cap \mathrm{A} \Rightarrow \neg \mathrm{C}$, which is contradicting).

I will further call this argumentation "superposed-action explanation". It is grounded on the assumption that Alice's and Bob's states (1) and (2) have evolved unitarily to a composite entangled state (8), after Alice handed the second state to Bob.

\section{Superposed Action}

Let us have a closer look on how the state (8) could possibly be created using the procedure of the EWF under the standard assumptions of Wigner's friend thought

${ }^{2}$ Of course the notation " $|-\rangle \Rightarrow|h\rangle$ ” means that measurement of $|-\rangle$ implies measurement of $|h\rangle$, etc. 
experiments. Namely, it is assumed that from Charlie's super-observer perspective, after the state (1) is measured by Alice at time $t_{0}$, it becomes a tensor product:

$$
\left.\left.\left|\alpha\left(t_{0}\right)\right\rangle=\sqrt{\frac{1}{3}}|h\rangle \mid \text { Alice knows } h\right\rangle+\sqrt{\frac{2}{3}}|t\rangle \mid \text { Alice knows } t\right\rangle .
$$

For the next stage of the EWF, a usual definition of the

"Freedom of Choice". The choice of measurement settings is statistically independent from the rest of the experiment (Statement 3 in the no-go theorem of Ref. [6]),

seems, however, insufficient. Probability amplitudes are fixed in all the states and bases of the EWF (they do not need to be chosen in each round of this thought experiment). Therefore, proposed, amended definition is:

"Freedom of Choice". Observer's opportunity and autonomy to prepare a quantum state in one of the two available nonorthogonal versions, wherein that state will be measured later by other observer (s), is statistically independent from the rest of the experiment.

There is no guarantee that an agent inside the lab will act as expected. And that makes it impossible to derive arguments that implicitly assume that she acts properly and reports valid measurement results.

We note in passing that such an amended Freedom of Choice assumption is not explicitly made in [2]. It is therefore another hidden assumption of this thought experiment among other hidden assumptions [11].

Therefore, the following tensor product, corresponding to (12):

$$
\begin{aligned}
\left|\beta\left(t_{1}\right)\right\rangle & \left.\neq \sqrt{\frac{1}{3}}|0\rangle \mid \text { Bob knows } 0 \text {, as Alice knew } h \text { and prepared "|0 " at time } t_{0}\right\rangle \\
& \left.+\sqrt{\frac{1}{3}}|0\rangle \mid \text { Bob knows } 0 \text {, as Alice knew } t \text { and prepared " }|+\rangle " \text { at time } t_{0}\right\rangle(13) \\
& \left.+\sqrt{\frac{1}{3}}|1\rangle \mid \text { Bob knows } 1 \text {, as Alice knew } t \text { and prepared " }|+\rangle \text { " at time } t_{0}\right\rangle
\end{aligned}
$$

cannot be valid for Bob measuring, at time $t_{1}$, the state (2) prepared by Alice at time $t_{0}$, as this would violate Alice's freedom of choice. The absence of observer-independent measurements [5] [6] allows one to discuss superpositions of observer-dependent measurements (12) of time-independent quantum states, but not to discuss superpositions of observer-dependent and time-dependent observer's actions (13).

An action of Alice is relative not only to Alice [5] [6]. It can be observed also by Bob and Charlie.

Therefore, from Charlie's super-observer perspective quantum register containing the first qubit (1), and the second state $|\beta\rangle=|0\rangle$ or $|\beta\rangle=(|0\rangle+|1\rangle) / \sqrt{2}$ after it was prepared by Alice would initially contain either two separable pure states $\{|h 0\rangle,|t 0\rangle\}$ (if Alice measured $|h\rangle)$ : 


$$
|\psi\rangle_{h 0}=\left(\sqrt{\frac{1}{3}}|h\rangle+\sqrt{\frac{2}{3}}|t\rangle\right) \otimes|0\rangle=\sqrt{\frac{1}{3}}|h 0\rangle+\sqrt{\frac{2}{3}}|t 0\rangle,
$$

or four separable pure states $\{|h 0\rangle,|h 1\rangle,|t 0\rangle,|t 1\rangle\}$ (if Alice measured $|t\rangle$ ):

$$
\begin{aligned}
|\psi\rangle_{t 01} & =\left(\sqrt{\frac{1}{3}}|h\rangle+\sqrt{\frac{2}{3}}|t\rangle\right) \otimes\left(\sqrt{\frac{1}{2}}|0\rangle+\sqrt{\frac{1}{2}}|1\rangle\right) \\
& =\sqrt{\frac{1}{6}}|h 0\rangle+\sqrt{\frac{1}{6}}|h 1\rangle+\sqrt{\frac{2}{6}}|t 0\rangle+\sqrt{\frac{2}{6}}|t 1\rangle
\end{aligned}
$$

The reader may be tempted at this point to question the validity of Equations (14) and (15). If the first qubit is measured (by Alice) it retains its state and instead of (14) and (15) we would have respectively $|h\rangle|0\rangle$ or $|t\rangle(|0\rangle+|1\rangle) / \sqrt{2}=|t\rangle|+\rangle$. The point here is that we consider three versions of the EWF. The $1^{\text {st }}$ one deals with just two states (1) and (2) that are not entangled and retain their states after measurements. This version is described by density matrices (5), (6) showing no contradiction. The $2^{\text {nd }}$ one [1] [2] assumes entanglement of these states along with the content of the Alice's lab. Although the author considers such an assumption as unrealistic, it is a priori assumed to be correct. However, in this setup Alice's action must be taken into account, as Bob's lab cannot be described by the state (13), which leads us to Equations (14), (15), and the $3^{\text {rd }}$ version of the EWF.

In order to affect the unitary evolution of such two different quantum registers to bring them both to the entangled state $|\psi\rangle_{A B}(8)$, that would be the same regardless of the initial state $|\psi\rangle_{h 0}$ (14) or $|\psi\rangle_{01}$ (15), Alice must use two different variants of some suitable $4 \times 4$ unitary matrices.

If she measures the state (1) as $|h\rangle$ she may use first the following unitary ma$\operatorname{trix} A_{h 0}$ :

$$
A_{h 0}|\psi\rangle_{h 0}=\left[\begin{array}{cccc}
\sqrt{\frac{1}{3}} & 0 & \sqrt{\frac{2}{3}} & 0 \\
0 & \sqrt{\frac{1}{3}} & 0 & -\sqrt{\frac{2}{3}} \\
\sqrt{\frac{2}{3}} & 0 & -\sqrt{\frac{1}{3}} & 0 \\
0 & \sqrt{\frac{2}{3}} & 0 & \sqrt{\frac{1}{3}}
\end{array}\right]\left[\begin{array}{c}
\sqrt{\frac{1}{3}} \\
0 \\
\sqrt{\frac{2}{3}} \\
0
\end{array}\right]=\left[\begin{array}{l}
1 \\
0 \\
0 \\
0
\end{array}\right]=|\psi\rangle_{00},
$$

while if she measures the state (1) as $|t\rangle$ she may use first the following unitary matrix $A_{t 01}$ :

$$
A_{t 01}|\psi\rangle_{t 01}=\left[\begin{array}{cccc}
\sqrt{\frac{1}{6}} & \sqrt{\frac{1}{6}} & \sqrt{\frac{1}{3}} & \sqrt{\frac{1}{3}} \\
\sqrt{\frac{1}{3}} & \sqrt{\frac{1}{3}} & -\sqrt{\frac{1}{6}} & -\sqrt{\frac{1}{6}} \\
-\sqrt{\frac{1}{6}} & \sqrt{\frac{1}{6}} & \sqrt{\frac{1}{3}} & -\sqrt{\frac{1}{3}} \\
\sqrt{\frac{1}{3}} & -\sqrt{\frac{1}{3}} & \sqrt{\frac{1}{6}} & -\sqrt{\frac{1}{6}}
\end{array}\right]\left[\begin{array}{l}
\sqrt{\frac{1}{6}} \\
\sqrt{\frac{1}{3}} \\
\sqrt{\frac{1}{3}}
\end{array}\right]=\left[\begin{array}{l}
1 \\
0 \\
0 \\
0
\end{array}\right]=|\psi\rangle_{00} .
$$


Then she may use the following unitary matrix $R$ :

$$
R|\psi\rangle_{00}=\left[\begin{array}{cccc}
\sqrt{\frac{1}{3}} & 0 & \sqrt{\frac{1}{2}} & \sqrt{\frac{1}{6}} \\
0 & 1 & 0 & 0 \\
\sqrt{\frac{1}{3}} & 0 & -\sqrt{\frac{1}{2}} & \sqrt{\frac{1}{6}} \\
\sqrt{\frac{1}{3}} & 0 & 0 & -\sqrt{\frac{2}{3}}
\end{array}\right]\left[\begin{array}{l}
1 \\
0 \\
0 \\
0
\end{array}\right]=\left[\begin{array}{c}
\sqrt{\frac{1}{3}} \\
0 \\
\sqrt{\frac{1}{3}} \\
\sqrt{\frac{1}{3}}
\end{array}\right]=|\psi\rangle_{A B},
$$

to receive $|\psi\rangle_{A B}=R|\psi\rangle_{00}$ from $|\psi\rangle_{00}=A_{h 0}|\psi\rangle_{h 0}$ or $|\psi\rangle_{00}=A_{001}|\psi\rangle_{001}$.

There are obviously infinitely many possibilities of unitary transformations that would bring $|\psi\rangle_{h 0}$ or $|\psi\rangle_{01}$ to $|\psi\rangle_{A B}$ (8), as groups of unitary $4 \times 4$ matrices act transitively on the unit vectors in Hilbert spaces over the complex numbers (4). The above exemplary forms model the evolution of the EWF, provided they are correctly used.

Indeed, such an approach assumes that in order to arrive at $|\psi\rangle_{A B}$ Alice must after recording the outcome of her measurement of the first qubit to be either $|h\rangle$ or $|t\rangle$ act according to this outcome in a manner predefined by (2) by applying an appropriate unitary matrix transformation.

But what if Alice makes a mistake and applies a "wrong" transformation $R A_{h 0}$ or $R A_{101}$, respectively to the quantum register $|\psi\rangle_{t 01}$ (15) or $|\psi\rangle_{h 0}$ (14)?

Assume, for example, that there is no agent (no Alice) performing the "actual" measurement of the first qubit (1) in Alice's isolated lab but just some mechanism applying randomly with a probability $p$ matrix transformation $R A_{h 0}$ and with the probability $(1-p)$ matrix transformation $R A_{001}$ to the initial separable state $|\psi\rangle_{h 0}$ or $|\psi\rangle_{t 01}$. Table 1 lists the results of such a mechanism operation.

In this scenario the state $|\psi\rangle_{A B}$ would be obtained with probability of $p / 3+2(1-p) / 3=2 / 3-p / 3$ which has maximum of $2 / 3$ for $p=0$. But the following two (at least $|\psi\rangle_{A B t h}$ for $p=0$ ) different states $|\psi\rangle_{A B t h}$ and $|\psi\rangle_{A B h t}$ would also be obtained:

$$
\begin{gathered}
|\psi\rangle_{A B t h}=0.847|h 0\rangle+0.514|t 0\rangle-0.136|t 1\rangle, \\
|\psi\rangle_{A B h t}=0.680|h 0\rangle-0.236|h 1\rangle+0.680|t 0\rangle-0.136|t 1\rangle,
\end{gathered}
$$

certainly with nonzero probability, if one assumes that Alice has the freedom of

Table 1. Random application of composite matrix transformations $R A_{h o}$ and $R A_{t o l}$.

\begin{tabular}{ccccc}
\hline $\begin{array}{c}\text { Initial } \\
\text { State }\end{array}$ & $\begin{array}{c}\text { Initial State } \\
\text { Probability }\end{array}$ & $\begin{array}{c}\text { Applied } \\
\text { Transformation } \\
\text { Probability }\end{array}$ & $\begin{array}{c}\text { Applied } \\
\text { Transformation and } \\
\text { the Resultant State }\end{array}$ & $\begin{array}{c}\text { Resultant } \\
\text { Probability }\end{array}$ \\
\hline \multirow{2}{*}{$\begin{array}{l}\text { Th }\rangle_{h 0} \\
\end{array}$} & $1 / 3$ & $p$ & $R A_{h 0}|\psi\rangle_{h 0}=|\psi\rangle_{A B}$ & $p / 3$ \\
& & $1-p$ & $R A_{001}|\psi\rangle_{h 0}=|\psi\rangle_{A B t h}$ & $(1-p) / 3$ \\
$|\psi\rangle_{01}$ & $2 / 3$ & $p$ & $R A_{h 0}|\psi\rangle_{01}=|\psi\rangle_{A B h t}$ & $2 p / 3$ \\
& & $1-p$ & $R A_{001}|\psi\rangle_{01}=|\psi\rangle_{A B}$ & $2(1-p) / 3$ \\
\hline
\end{tabular}


choice in affecting the unitary evolution of the initial state $|\psi\rangle_{h 0}$ (14) or $|\psi\rangle_{01}$ (15). And Alice's freedom of choice implies her fallibility. These states in mixed bases $\{|h\rangle,|t\rangle\},\{|0\rangle,|1\rangle\} ;\{|\square\rangle,|O\rangle\},\{|+\rangle,|-\rangle\}(3),(4)$ are for $|\psi\rangle_{A B t t}$ :

$$
\begin{gathered}
|\psi\rangle_{C t h}=0.612|\square+\rangle+0.749|\square-\rangle+0.235|\bigcirc+\rangle+0.099|\bigcirc-\rangle, \\
|\psi\rangle_{\text {ACth }}=0.599|h+\rangle+0.599|h-\rangle+0.267|t+\rangle+0.460|t-\rangle, \\
|\psi\rangle_{\text {CBth }}=0.962|\square 0\rangle+0.236|\bigcirc 0\rangle-0.096|\square 1\rangle+0.096|\bigcirc 1\rangle,
\end{gathered}
$$

and for $|\psi\rangle_{A B h t}$

$$
\begin{gathered}
|\psi\rangle_{C h t}=0.495|\square+\rangle+0.866|\square-\rangle-0.050|\bigcirc+\rangle+0.050|\bigcirc-\rangle, \\
|\psi\rangle_{A C h t}=0.315|h+\rangle+0.648|h-\rangle+0.385|t+\rangle+0.577|t-\rangle, \\
|\psi\rangle_{C B h t}=0.962|\square 0\rangle-0.263|\square 1\rangle-0.070|\bigcirc 1\rangle .
\end{gathered}
$$

Quantum-cakes-explanation fails both for $|\psi\rangle_{A B t h}$ and $|\psi\rangle_{A B h t}\left({ }^{*}\right.$ denotes th or $h t)$ :

(\#1) We know from $|\psi\rangle_{C^{*}}(21)$, (24) that measurements of $\left|\mathrm{O}_{-}\right\rangle$are possible;

(\#2) We know from $|\psi\rangle_{A C^{*}}(22)$, (25) that $|-\rangle \nRightarrow|h\rangle$ (unlike $|\psi\rangle_{A C}(10)$ ).

Superposed-action explanation also fails, if Alice makes a mistake. Charlie can measure $|\psi\rangle_{C^{+}}$at time $t_{2}$ and observe $\left|O_{-}\right\rangle$but Alice's claim based on (2) that $|t\rangle$ measured at time $t_{0}$ implies $(|0\rangle+|1\rangle) / \sqrt{2}=|+\rangle$ measured at time $t_{2}$ is false merely due to her own mistake. Neither Bob's claim, also based on (2), that $|1\rangle$ measured at time $t_{1}$ implies $|t\rangle$ measured at time $t_{0}$ is true.

Elementary arithmetic shows that any normalized nonmaximally entangled state:

$$
|\psi\rangle_{A B}=a|h 0\rangle+c|t 0\rangle+d|t 1\rangle,
$$

measured in the EWF bases is:

$$
\begin{gathered}
|\psi\rangle_{C}=\frac{a+c+d}{2}|\square+\rangle+\frac{a+c-d}{2}|\square-\rangle+\frac{a-c-d}{2}|\bigcirc+\rangle+\frac{a-c+d}{2}|\bigcirc-\rangle, \\
|\psi\rangle_{A C}=\frac{a}{\sqrt{2}}(|h+\rangle+|h-\rangle)+\frac{c+d}{\sqrt{2}}|t+\rangle+\frac{c-d}{\sqrt{2}}|t-\rangle, \\
|\psi\rangle_{C B}=\frac{a+c}{\sqrt{2}}|\square 0\rangle+\frac{a-c}{\sqrt{2}}|\bigcirc 0\rangle+\frac{d}{\sqrt{2}}(|\square 1\rangle-|\bigcirc 1\rangle),
\end{gathered}
$$

where the normalization constraint $(\langle\psi \mid \psi\rangle=1)$ and the surjective isometry constraints: $c-d=0$ in (29) to suppress $|t-\rangle$, and $a-c=0$ in (30) to suppress $|O 0\rangle$, imposed to satisfy the conditions (\#2), (\#2') of the quantum-cakes explanation, lead to the unique solution of $a=c=d$ with probability amplitude having modulus $|d|=1 / \sqrt{3} .|\psi\rangle_{A B t h}(19)$ and $|\psi\rangle_{A B h t}(20)$ do not represent this solution.

Therefore, the entangled quantum state that Alice delivers to Bob may not be $|\psi\rangle_{A B}$ (8) but $|\psi\rangle_{A B t h}$ (19) and $|\psi\rangle_{A B h t}(20)$ as well, regardless of the outcome of her measurement of the first qubit (1). The contradiction of the EWF cannot be discussed in isolation from Alice's freedom of choice understood as her fallibility. 


\section{Conclusion}

Authors of the EWF argue to have arrived at the contradiction by letting the second state (2), which Bob receives from Alice, to depend on a random value measured and known by Alice [2]. But since there is no unitary transformation that would bring a pure, single qubit state (1) and two nonorthogonal versions of a mixed state (2) into an entangled, pure, two-qubit state (8), as discussed above, this argumentation is false.

\section{Discussion}

The legitimacy of the EWF procedure has been questioned [14] [15], respectively under a nonunitary account of quantum state reduction, and decoherence. Indeed, the measurement problem is the only source of contradictions in Bell-type experiments. But in the case of the EWF, it is not the incompatibility of the unitary evolution and the measurement involved in the piecewise-defined function (2) that is not self-consistent [16] [17], but this nonorthogonal piecewise definition, as such. In this particular 1 out of 12 (on average) rounds of the experiment where $\left|\mathrm{O}^{-}\right\rangle$is measured by Charlie the if-and-only-ifs in (2) cannot be guaranteed to hold and possible errors of Alice must be accounted for.

The presence of these errors should discourage a casino manager from offering a gambling game based on the principles of the EWF. In a dispute between a gambler and a casino, the judge should rule in favor of the gambler: shifting the responsibility for the erroneous operation of this game to the gambler (consumer) appears to be an unfair commercial practice. Fortunately, such considerations are academic and no judge will ever need to rule in such a case as the EWF is impossible to be implemented as a game in a casino. Unitary transformations of the big quantum state (8) performed from within the lab by Alice herself [2] pursuant to the recipes of (1) and (2), or similar, are impossible.

Numerous other publications (e.g., [18] [19] [20]) attempt to explain the EWF using Bohmian mechanics. But Bohmian interpretation of QT is incompatible with the assumptions of (i) the choice of which measurement is performed can be made randomly and independently of the system under observation, (ii) the system has limited memory, and (iii) Landauer's erasure principle holds [21]. Assumption (i) is the standard "Freedom of Choice" assumption of Bell-type experiments (freedom of what to measure), while assumptions (ii) and (iii) form the basis of the prevailing explanation of Maxwell demon (Szilard's engine) paradox. The author sees no reason to question these assumptions. "Pilot-wave theories are parallel-universe theories in a state of chronic denial" [22], while the aim of this paper is to save the single world, rather than delve into multiverses that cannot be experimentally confirmed.

Much of the essence of QT already makes itself known in the case of just two nonorthogonal states [23]. But in the case of the EWF, the specific type of quantum states (1), (2), (8), measurements, outcomes and actions involved in the argument are relevant and should not be omitted [6]. This is important if one com- 
pares the EWF with Bell-type experiments, in particular with those belonging to their subset, which excludes the coexistence of observer-independent measurements [5] [6] [7] [8] (termed as Bell-Wigner type experiments in Ref. [7]). Observer-independent measurements do not exist [5] [6] but this conclusion cannot be derived through the backdoor. It manifests in collected statistics of measurements of a specific, entangled state, but not in the flawed argument of a superposed-action.

On the contrary to the EWF, a casino manager should not be discouraged from offering a gambling game based on the principles of the Bell-Wigner type experiments. They are by all means implementable in practice, while errors are relatively small. 6-photon Bell-Wigner type experiment violated the associated Bell-type inequality by 5 standard deviations [7]. But here Alice, Bob and Charlie are photon detectors whose detections are processed by a classical computer to find 6-photon coincidence events. Thus Alice and Bob not only inform Charlie about obtaining definite measurement results [10] (using heralding signals $\alpha$ and $\beta$ ) but also reveal these results, and yet do not destroy the superposition of the entangled state. As Bell-Wigner type experiments boil down to nonlocal correlations of observer-dependent measurements, which correlations are known at least since Bells' remarkable theorem, a judge would be presented with an easy task in any dispute between a gambler and a casino: lack of observer-independent measurements [5] [6] is a known, experimentally proven [7] [8] feature of QT. Different measurement times could be easily introduced in Bell-Wigner type setups. In the case of photonic implementation, physical delays can be employed on particular light guides between a laser and detectors. On the other hand, in a relativistic frame of reference of a photon, no time passes between the emission of the photon and its absorption, which is otherwise known as time dilation. A quantum state (in particular an entangled one) is time-independent.

But what is the Single World? The Single World is the world of a single observ$\mathrm{er}^{3}$. There are at least about 7.9 billion single worlds (as of November 2021; restricting observers to humans able to communicate their observations in abstract terms).

\section{Acknowledgements}

I truly thank my wife for her support and Mirek for encouragement.

\section{Conflicts of Interest}

The author declares no conflicts of interest regarding the publication of this paper.

\section{References}

[1] Renner, D.F.R. (2016) Single-World Interpretations of Quantum Theory Cannot Be Self-Consistent.

[2] Renner, D.F.R. (2018) Quantum Theory Cannot Consistently Describe the Use of

“"So many stars, so many ways of seeing” (U2). 
Itself. Nature Communications, 9, Article No. 3711. https://doi.org/10.1038/s41467-018-05739-8

[3] Elitzur, A.C. and Vaidman, L. (1993) Quantum Mechanical Interaction-Free Measurements. Foundations of Physics, 23, 987-997.

https://doi.org/10.1007/BF00736012

[4] Blaylock, G. (2010) The EPR Paradox, Bell's Inequality, and the Question of Locality. American Journal of Physics, 78, 111-120. https://doi.org/10.1119/1.3243279

[5] Brukner, Č. (2015) On the Quantum Measurement Problem. Springer, Cham. https://doi.org/10.1007/978-3-319-38987-5 5

[6] Brukner, Č. (2018) A No-Go Theorem for Observer-Independent Facts. Entropy, 20, 350. https://doi.org/10.3390/e20050350

[7] Proietti, M., et al. (2019) Experimental Test of Local Observer Independence. Science Advances, 5, eaaw9832. https://doi.org/10.1126/sciadv.aaw9832

[8] Bong, K.-W., Utreras-Alarcon, A., Ghafari, F., Liang, Y.-C., Tischler, N., Cavalcanti, E.G., Pryde, G.J. and Wiseman, H.M. (2019) Testing the Reality of Wigner's Friend's Experience. https://doi.org/10.1117/12.2540002

[9] Wigner, E. (1961) The Scientist Speculates. 284-302.

[10] Deutsch, D. (1985) Quantum Theory as a Universal Physical Theory. International Journal of Theoretical Physics, 24, 1-41. https://doi.org/10.1007/BF00670071

[11] Sudbery, A. (2019) The Hidden Assumptions of Frauchiger and Renner. The International Journal of Quantum Foundations, 5, 98.

[12] Bub, J. (2017) Why Bohr Was (Mostly) Right.

[13] Kwiat, P. and Hardy, L. (2000) The Mystery of the Quantum Cakes. American Journal of Physics, 68, 33-36. https://doi.org/10.1119/1.19369

[14] Kastner, R.E. (2020) Unitary-Only Quantum Theory Cannot Consistently Describe the Use of Itself: On the Frauchiger-Renner Paradox. Foundations of Physics, 50, 441-456. https://doi.org/10.1007/s10701-020-00336-6

[15] Shuler, R.L. (2021) Emergence of Objective Reality in an Irreversible Friend Thought Experiment. Journal of Applied Mathematics and Physics, 9, 1964-1977. https://doi.org/10.4236/jamp.2021.98128

[16] Sudbery, A. (2017) Single-World Theory of the Extended Wigner's Friend Experiment. Foundations of Physics, 47, 658-669. https://doi.org/10.1007/s10701-017-0082-7

[17] Baumann, V., Hansen, A. and Wolf, S. (2016) The Measurement Problem Is the Measurement Problem Is the Measurement Problem.

[18] Lazarovici, D. and Hubert, M. (2019) How Quantum Mechanics Can Consistently Describe the Use of Itself. Scientific Reports, 9, Article No. 470.

https://doi.org/10.1038/s41598-018-37535-1

[19] Muciño, R. and Okon, E. (2020) Wigner's Convoluted Friends. Studies in History and Philosophy of Science Part B: Studies in History and Philosophy of Modern Physics, 72, 310. https://doi.org/10.1016/j.shpsb.2020.07.001

[20] Tausk, D.V. (2018) A Brief Introduction to the Foundations of Quantum Theory and an Analysis of the Frauchiger-Renner Paradox.

[21] Cabello, A., Gu, M., Gühne, O., Larsson, J.-Å. and Wiesner, K. (2016) Thermodynamical Cost of Some Interpretations of Quantum Theory. Physical Review A, 94, Article ID: 052127. https://doi.org/10.1103/PhysRevA.94.052127

[22] Deutsch, D. (1996) Comment on Lockwood. British Journal for the Philosophy of Science, 47, 222-228. https://doi.org/10.1093/bjps/47.2.222 
[23] Fuchs, C.A. (1998) Just Two Nonorthogonal Quantum States. Quantum Communication, Computing, and Measurement, 2, 11-16.

https://doi.org/10.1007/0-306-47097-7 2 